\title{
Are we there yet?
}

\author{
Gráinne Fadden
}

\section{COMMENTARY ON... FAMILY THERAPY AND SCHIZOPHRENIA}

\begin{abstract}
SUMMARY
Burbach describes the content of a phased approach to delivering family work in psychosis. Clinicians would find it helpful to have guidance on how to address the challenges they face in clinical practice, such as engaging all family members in the process and how to deal with confidentiality conflicts. Implementation challenges are also likely to affect their ability to deliver this intervention. It may also be useful to consider the role that family members can play in co-production and training, and in delivering support to other families through a carer peer support model.
\end{abstract}

\section{DECLARATION OF INTEREST}

None.

There have been substantial developments in how family work is delivered in current clinical practice, and a clear rapprochement between different family work models. Although family work is recommended in the National Institute for Health and Care Excellence guidelines for psychosis and family members in the UK have a legal entitlement to have their needs assessed, implementation of family work remains problematic. Burbach (2018, this issue) provide a useful summary of contemporary family work for people with psychosis. It is helpful in conveying how family approaches have evolved and developed over time, with the old divisions between systemic family therapy and the more psychoeducational approaches becoming blurred. The flexibility of current practice is conveyed well, as is the current pragmatic and positive style of delivering these approaches. There is a good section on cultural issues, emphasising the fact that the respectful, psychologically curious and non-dogmatic nature of these approaches means that they are acceptable across cultures. The variation in what different families require is well conveyed, and the value of even brief interventions is highlighted. The extensive clinical experience and expertise of the author is evident throughout.

The article well describes the different phases of a brief family intervention and what the intervention looks like. In a way, this feels like the first step the 'what' of family work. Here, I address relevant areas that are linked with clinical and organisational factors, more related to the next step - the 'how to'.

\section{Clinical considerations}

There are many challenges that clinicians may struggle with in relation to delivering family interventions (Bucci 2015), and guidance on the most common of these would be helpful. These include the following questions: Who should decide if family work is appropriate for this family? What if the patient does not want to engage in the sessions but other family members do? Can cognitive therapy for the patient and family work be delivered at the same time? Who should deliver family work, the care coordinator or someone else? At what point should family work start? Where should sessions be delivered?

The issue of confidentiality is one of the most challenging for clinicians delivering family work, and one on which guidance is frequently sought (Gold 2009). The following is an example of the kind of practical guidance clinicians value to help them to deal with confidentiality conflicts.

It is generally helpful to start to have conversations with the patient about their concerns, for example, what are they worried about? Is there certain information they wish to remain private? What might be the benefits for them if their family understood more about what they are experiencing? There are many helpful articles on the topic of confidentiality that family workers can be guided to, such as Rapaport (2006) and Wilson (2015).

\section{Organisational considerations}

\section{Supervision}

Supervision is an area that I would have liked to see emphasised more in the article, as it is essential for anyone practising family work in order to address the challenges faced and to ensure safe practice (Eassom 2014). It is essential not only at a clinical level, but is also one of the factors that facilitates implementation of family work at an organisational level.
Gráinne Fadden is the director of the Meriden Family Programme at the Birmingham and Solihull Mental Health NHS Foundation Trust, UK. His areas of interest/research include the implementation of family work and recovery for families.

Correspondence Dr Gráinne Fadden, Meriden Family Programme, Tall Trees, Uffculme Centre, Queensbridge Road, Birmingham B13 80Y, UK. Email: grainne.fadden1@nhs.net

\section{Copyright and usage}

(C) The Royal College of Psychiatrists 2018 


\section{Service issues}

Although there is a very brief section on service considerations, we have known for 25 years, since Kavanagh (1993) first highlighted the issue, that the biggest challenge in relation to family interventions is their widespread dissemination in practice. There have been publications outlining barriers to implementation (Berry 2008; Bucci 2015), articles looking at factors facilitating implementation (Bailey 2003; Fadden 2011) and an excellent systematic review of both facilitating and hindering factors (Eassom 2014).

Anyone attempting to deliver family interventions is likely to experience setbacks, so an awareness of the challenges and what helps to overcome them will make it less likely that they will become despondent and give up. Barriers to implementation include lack of training and supervision, failure of the organisation to prioritise family work, lack of ring-fenced time and attitudinal barriers (Bucci 2015). Facilitating factors include strong leadership and management support, developing a culture that is positive towards family work delivery, creating roles specifically for delivering family work or having family work champions, training and supervision, making family work part of routine clinical work and engaging with family members as equal partners (Eassom 2014). There is some evidence that there is less resistance to the delivery of family work than in the past, and that there has been some attitudinal shift even if the delivery of family work is still problematic (Selick 2017).

\section{Future directions}

Technological options to delivering family work are a possible direction for the future, and are mentioned in the article. Self-help options can also be relevant for family members who find it difficult to have time away from the home because of their caring responsibilities; for example, a 'Caring for Yourself' manual (Fadden 2012).

The article clearly has a recovery focus, and the concept of recovery for family members is developing, giving rise to interesting question such as those related to co-recovery (Fox 2015). For example, can family members attend to their own recovery if the patient remains unwell?

There are helpful developments around co-production (Bradley 2015) and carer peer support (Craddock 2013; Bourke 2015). It would be interesting to consider how increased involvement with family members as partners in delivering care would affect implementation challenges. This could potentially be a valuable addition to the workforce in areas where there are capacity issues.

\section{References}

Bailey R, Burbach F, Lea S (2003) The ability of staff trained in family interventions to implement the approach in routine clinical practice. Journal of Mental Health, 12(2): 131-41.

Berry K, Haddock G (2008) The implementation of the NICE guidelines for schizophrenia: barriers to the implementation of psychological interventions and recommendations for the future. Psychology and Psychotherapy, 81(4): 419-36.

Bourke C, Sanders B, Allchin B, et al (2015) Occupational therapy influence on a carer peer support model in a clinical mental health service. Australian Occupational Therapy Journal, 62: 299-305.

Bradley E (2015) Carers and co-production: enabling expertise through experience? Mental Health Review Journal, 20: 232-41.

Bucci S, Berry K, Barrowclough C, et al (2015) Family Interventions in psychosis: a review of the evidence and barriers to implementation. Australian Psychologist, 51: 62-8.

Burbach FR (2018) Family therapy and schizophrenia: a brief theoretical overview and a framework for clinical practice. BJPsych Advances, 24: 225-34.

Craddock E (2013) Supporting mental health carers' role in recovery. Nursing Times, 109: 22-4.

Eassom E, Giacco D, Dirik A, et al (2014) Implementing family involvement in the treatment of patients with psychosis: a systematic review of facilitating and hindering factors. BMJ Open, 4: e006108.

Fadden G, Heelis R (2011) The Meriden West Midlands family programme: lessons learned over ten years. Journal of Mental Health, 20 (1): 79-88.

Fadden G, James C, Pinfold V (2012) Caring for Yourself. Rethink Mental Illness and Meriden Family Programme, 2012. (https://www.meriden familyprogramme.com/recovery/what-carers-families-and-friends-can-doto-help-themselves/caring-for-yourself).

Fox J, Ramon S, Morant N (2015) Exploring the meaning of recovery for carers: implications for social work practice. British Journal of Social Work, 45(suppl 1): i117-34.

Gold M, Philip J, Mclver S, et al (2009) Between a rock and a hard place: exploring the conflict between respecting the privacy of the individual and informing their carers. Internal Medicine Journal, 39: 357-65.

Kavanagh DJ, Piatkowska O, Clarke D, et al (1993) Application of cognitive-behavioural family intervention for schizophrenia in multi-disciplinary teams: what can the matter be? Australian Psychologist, 28: 181-8.

Rapaport J, Bellringer S, Pinfold V, et al (2006) Carers and confidentiality in mental health: considering the role of the carer's assessment: a study of service users', carers' and practitioners' views. Health and Social Care in the Community, 14(4): 357-65.

Selick A, Durbin J, Vu N, et al (2017) Barriers and facilitators to implementing family support and education in early psychosis intervention programmes: a systematic review. Early Intervention in Psychiatry. 11(5): 365-74.

Wilson LS, Pillay D, Kelly BD, et al (2015) Mental health professionals and information sharing: carer perspectives. International Journal of Medical Science. 184(4): 781-90. 